\title{
E-cigarettes and smoking cessation
}

\author{
Menfil Orellana-Barrios MD, Drew Payne DO
}

Electronic cigarettes (e-cigarettes) are an increasingly popular source of nicotine for the general public. Their advertising, commercialization, and use have been rising since their introduction into the US market in 2007. Also, the medical literature on the topic of e-cigarettes has dramatically increased from 48 PubMed citations in 2010 to $>1,600$ currently. Clinicians should be aware of the potential health effects of e-cigarettes since patients' interest, use, and questions related to electronic nicotine delivery devices occur increasingly during clinic visits. Common scenarios a clinician may face are: current smokers seeking a method of smoking cessation/reduction, current smokers seeking an additional source of nicotine (socalled dual use), and never smokers interested in trying out these novel devices.

E-cigarettes have obvious health implications, but current US and even worldwide regulations are scarce and a source of scientific and political controversy. Another factor complicating the debate is that e-cigarettes vary in structure and function as does the content of the e-cigarette solution. For example, the battery potency of each individual model affects the aerosol (colloquially termed "vapor", hence the term "vaping") produced and the types of e-cigarettes solutions are numerous. Most e-cigarette solutions, a.k.a. "e-juice", contain propylene glycol, flavoring, and nicotine in varying concentrations, but some are nicotine-free and therefore would require different regulation than those with nicotine. These arguments can get complicated quite quickly. We consider the main topics of discussion as:

1. Can e-cigarettes serve as a smoking cessation aid?

2. Is there a role for e-cigarettes for harm reduction in smokers?

3. Are e-cigarettes a potential gateway to initiating smoking, particularly in young populations?
4. Should e-cigarettes be regulated as tobacco products?

5. What are the short and long term health effects of the inhalation of the aerosolized nicotine/nonnicotine solutions?

Several reviews ${ }^{1,2}$ on e-cigarettes are available to the interested reader. However, we would like to discuss some aspects of questions 1 and 2, given that current tobacco smoking is the leading source of preventable morbidity and mortality and produces a huge economic burden worldwide.

One major limitation in determining the utility of e-cigarettes as a smoking cessation aid is the very small number of randomized controlled clinical trials (RCTs) that actually compare e-cigarettes to other cessation methods. In a recent literature search on this topic, we reviewed 1,225 citations from PubMed and Web of Science (January 2007 to August 2015) and identified only three RCTs and six observational prospective studies. Only the ASCEND ${ }^{3}$ study (A Study of Smoking Cessation with Electronic Nicotine Devices; $\mathrm{N}=657$ ) has directly compared e-cigarettes with other cessation methods (nicotine patches) and reported that verified continuous abstinence at six months was highest in the nicotine e-cigarettes group (7.3\%), followed by the nicotine patches group (5.8\%), and nicotine-free e-cigarettes group (4.1\%). Both the ASCEND $^{3}$ and the ECLAT ${ }^{4}$ (EffiCiency and Safety of an eLectronic cigAreTte; $\mathrm{N}=300$ ) studies compared e-cigarettes with and without nicotine. In the ECLAT ${ }^{4}$ study, there were three groups: the first arm used 7.5 $\mathrm{mg} / \mathrm{mL}$ nicotine cartridge concentration; the second arm used $7.2 \mathrm{mg} / \mathrm{mL}$ cartridges for six weeks then 5.4 $\mathrm{mg} / \mathrm{mL}$ cartridges for another six weeks; and the third arm used nicotine-free cartridges. The 12 month abstinence rates for the three arms studied were $13 \%$, $9 \%$, and $4 \%$, respectively. The results of the third $\mathrm{RCT}$ are difficult to compare since there was no con- 
trol group without e-cigarette use and it had a smaller sample size ( $\mathrm{N}=48)$. In this study, the combined eight month abstinence rate for the e-cigarette groups was $19 \%(6 / 32)$, and nine participants (28\%) were lost to the follow up.

To date, there is inadequate evidence to conclude that e-cigarettes with nicotine have a higher cessation rate than e-cigarettes without nicotine. $\mathrm{A}$ much bigger question is whether or not e-cigarettes, compared to other methods, are effective cessation devices. Interestingly, most smokers who actually quit do so without nicotine replacement, medication, or counseling, and the research on unassisted smoking cessation is also scarce. ${ }^{6}$ Therefore, more studies of e-cigarettes versus placebo and other methods (e.g., other forms of NRTs, counseling, medications) are needed to evaluate the true impact of e-cigarettes.

E-cigarettes have a definite potential for harm from acute health effects and unknown long term effects. At the same time, despite all the politics, there is undeniable potential for benefit in terms of smoking harm reduction. Clinicians are faced with difficult to answer questions regarding e-cigarettes but simply explaining to patients the current knowledge (or lack thereof) and letting the patient make an informed decision is the best we can do for now.

Author Affiliation: Menfil Orellana-Barrios and Drew Payne are residents in Internal Medicine at Texas Tech University Health Sciences Center in Lubbock, TX.

Received: $12 / 07 / 2015$

Accepted: 12/19/2015

Published electronically: 01/15/2016

Conflict of Interest Disclosures: none

Corresponding Author : Menfil Orellana-Barrios MD

Contact Information: : Ma.orellana-barrios@ttuhsc.edu

DOI: $10.12746 / s w r c c c 2016.0413 .165$

\section{REFERENCES}

1. Orellana-Barrios MA, Payne D, Mulkey Z, Nugent K. Electronic cigarettes-a narrative review for clinicians. Am J Med. 2015;128(7):674-681.

2. Grana R, Benowitz N, Glantz SA. E-cigarettes a scientific review. Circulation. 2014;129(19):1972-1986.

3. Bullen $\mathrm{C}$, Howe $\mathrm{C}$, Laugesen $\mathrm{M}$, et al. Electronic cigarettes for smoking cessation: a randomised controlled trial. Lancet. 2013;382(9905):1629-1637.

4. Caponnetto P, Campagna D, Cibella F, et al. EffiCiency and Safety of an eLectronic cigAreTte (ECLAT) as tobacco cigarettes substitute: A Prospective 12-Month Randomized Control Design Study. Plos One. 2013;8(6).

5. McRobbie H, Bullen C, Hartmann-Boyce J, Hajek P. Electronic cigarettes for smoking cessation and reduction. Cochrane Database Syst Rev. 2014;12:Cd010216.

6. Chapman S, Wakefield MA. Large-scale unassisted smoking cessation over 50 years: lessons from history for endgame planning in tobacco control. Tob Control. 2013;22 Suppl 1:i33-35. 